\title{
Liquid Metals as alternative solution for the Power Exhaust of Future Fusion Devices: Status and Perspective
}

\author{
J.W. Coenen ${ }^{1}$, D.C.M.Van den Bekerom ${ }^{2}$,G. De Temmerman ${ }^{2}$, \\ G.Federici $^{3}$,V.Philipps ${ }^{1}$, G.Sergienko ${ }^{1}$, G.Strohmayer $^{4}$, \\ A.Terra ${ }^{1}$, B.Unterberg ${ }^{1}$, T.Wegener ${ }^{1}$ \\ ${ }^{1}$ Institute of Energy and Climate Research - Plasma Physics, Forschungszentrum \\ Jülich GmbH, Association EURATOM-FZJ, Partner In the Trilateral Euregio \\ Cluster, Jülich, Germany \\ ${ }^{2}$ FOM Institute DIFFER, Dutch Institute for Fundamental Energy Research, \\ Trilateral Euregio Cluster, The Netherlands \\ ${ }^{3}$ EFDA Power Plant Physics \& Technology, Garching, Germany \\ ${ }^{4}$ Max-Planck-Institut fuer Plasmaphysik, Garching Germany \\ E-mail: j.w.coenen@fz-juelich.de
}

\begin{abstract}
Applying liquid metals as Plasma Facing Components for fusion powerexhaust can potentially ameliorate lifetime issues as well as limitations to the maximum allowed surface heat loads by allowing for a more direct contact with the coolant. The material choice has so far been focused on lithium ( $\mathrm{Li}$ ) as it showed beneficial impact on plasma operation. Here materials such as tin $(\mathrm{Sn})$, gallium $(\mathrm{Ga})$ and aluminum $(\mathrm{Al})$ are discussed as alternatives potentially allowing higher operating temperatures without strong evaporation. Power loads of up to $25 \mathrm{MW} / \mathrm{m}^{2}$ for a $\mathrm{Sn} / \mathrm{W}$ component can be envisioned based on calculations and modeling. Reaching a higher operating temperature due to material re-deposition will be discussed. Liquids are typically facing stability issues due to $j \times B$ forces, potential pressure and MHD driven instabilities. The Capillary Porous System is used for stabilization by a mesh (W,Mo) substrate and replenishment by means of capillary action.
\end{abstract}




\section{Introduction}

A future fusion reactor faces several issues related to materials used in its construction. Among those are the issues related to the first wall and divertor surfaces, their power handling capabilities and lifetime. For the next generation device, ITER, a solution based on actively cooled tungsten (W) components has been developed for the divertor, while beryllium is used on the first wall [1]. For the next step devices, e.g. DEMO, or a future fusion reactor the limits on power-exhaust, availability and lifetime are quite stringent. Radiation effects including neutron embrittlement may limit actively cooled W components in DEMO to about 3-5 MW/ $\mathrm{m}^{2}$. Erosion of the 1 st Wall and the divertor will in addition require a significant armor thickness or short exchange intervals, while high-power transients need strong mitigation efficiency to prevent PFC damage [2]. Liquid metals have long been suggested to partly ameliorate life-time and power-exhaust issues by allowing for a self healing, self replenishing surface with no susceptibility to neutron damage. Several studies were dedicated to this topic $[3,4,5,6]$ as several tokamak facilities have established liquid metal related research. Typically the research focuses on liquid $\mathrm{Li}$ due to its low melting point and beneficial behavior on plasma performance $[7,8,9,10,11]$. In order to allow for stable operation the liquid is to be confined within a mesh or porous structure as already demonstrated in the devices T11, T10 [12], FTU [13] and NSTX [14]. In this contribution the potential operational limitations of the so called Capillary Porous Systems (CPS) [15, 16] and their application with materials other than Li are described. The CPS and its potential application under tokamak conditions as well as its heat-exhaust capabilities with respective liquid metal choices are studied. In addition experiments related to material compatibility, wetting as well as plasma impact are shown. Tin (Sn) due to its low melting point and low vapor pressure is the main candidate next to gallium (Ga). Aluminum ( $\mathrm{Al}$ ) is the least known material with respect to use as PFC.

\section{Liquid Metals - General Parameters}

As visible from table 1 one can already group the selected metals in three groups with their respective boiling point. $\mathrm{Li}$ as well as $\mathrm{W}$ are used as reference points.

\begin{tabular}{l||ccc|c||c}
\hline & $\mathrm{Sn}$ & $\mathrm{Ga}$ & $\mathrm{Al}$ & $\mathrm{Li}$ & $\mathrm{W}$ \\
\hline Melting Point $[\mathrm{K}]$ & 505 & 302 & 933 & 453 & 3695 \\
Boiling Point $[\mathrm{K}]$ & 2875 & 2477 & 2792 & 1615 & 5828 \\
$\rho\left[\mathrm{kg} \mathrm{m}^{-3}\right]$ & 7300 & 5910 & 2700 & 534 & 19250 \\
$\kappa\left[\mathrm{W} \mathrm{m}^{-1} \mathrm{~K}^{-1}\right]$ & 40 & 55 & 240 & 45 & 173 \\
$c\left[\mathrm{~J} \mathrm{~kg}^{-1} \mathrm{~K}^{-1}\right]$ & 250 & 380 & 990 & 4300 & 140 \\
\hline
\end{tabular}

Table 1: Material Properties [17] at RT

With $\mathrm{W}$ representing the material with the highest melting and boiling point, Li 
the lowest and $\mathrm{Sn}, \mathrm{Ga}$ and $\mathrm{Al}$ in an intermediate range. All selected candidate materials ( $\mathrm{Sn}, \mathrm{Ga}, \mathrm{Al}, \mathrm{Li}$ ) are liquid for the operating temperatures of the divertor surfaces with $\mathrm{Al}$ posing a challenge in terms of keeping it molten in piping and reservoirs.

Apart from sputter-erosion as known for solid PFCS evaporation needs to be considered for use of liquid metal PFCs. ([17] cf fig. 1(a)). Depending on the impurity

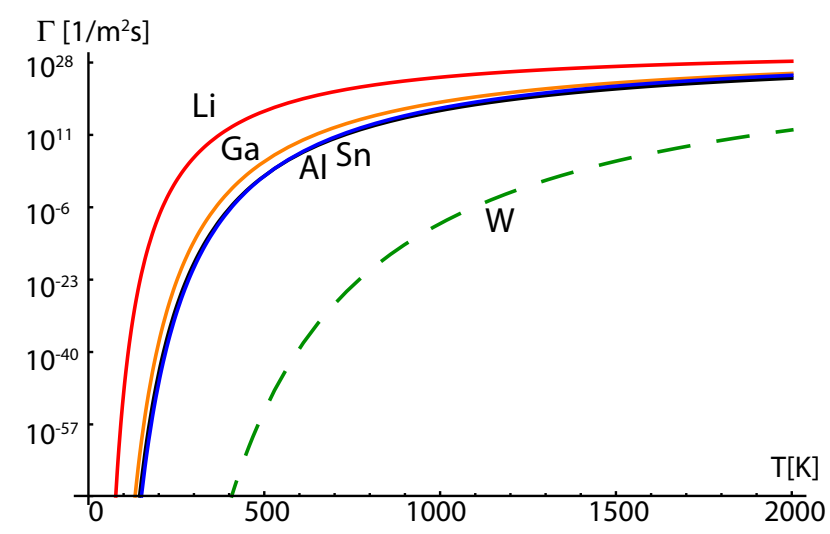

(a) Evaporation fluxes for different PFCs materials

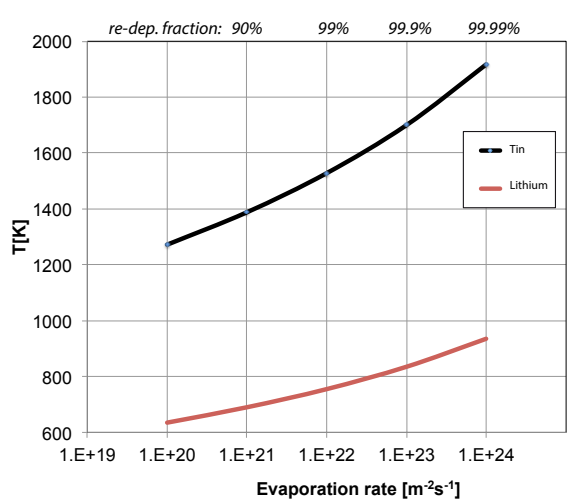

(b) Operational window considering redeposition

Figure 1: Typical Evaporative Fluxes for the considered liquid metal candidates. (a) displays the temperature dependence, while (b) is comparing $\mathrm{Sn}$ and Li with respect to potential re-deposition fractions.

and its atomic number $(\mathrm{Z})$ the influence on the plasma can be quite different ranging from fuel dilution of the fusion plasma (low-Z) to radiative collapse (high-Z) [18, 19]. A limit on the material flux needs to be set. One can define the limits with respect to the incoming plasma flux $\left(\sim 10^{24} \mathrm{~m}^{-2} s^{-1}\right)$. Considering typical sputter yields $(0.1-1 \%[20])$ an evaporation flux limit of at maximum $10^{22} \mathrm{~m}^{-2} s^{-1}$ seems a good starting point for the

\begin{tabular}{|c|c|c|c|}
\hline & $\mathrm{T}_{\min }[K]$ & $\mathrm{T}_{\max }[\mathrm{K}]\left(\Gamma_{E v .} / \Gamma_{P l}=10^{-4}\right)$ & $\mathrm{T}_{\max }[\mathrm{K}]\left(\Gamma_{E v .} / \Gamma_{P l}=10^{-2}\right)$ \\
\hline $\mathrm{Al}$ & 933 & 1200 & 1450 \\
\hline $\mathrm{Ga}$ & 303 & 1100 & 1300 \\
\hline $\mathrm{Sn}$ & 506 & 1272 & 1528 \\
\hline $\mathrm{Li}$ & 454 & 635 & 755 \\
\hline
\end{tabular}

Table 2: Operational surface temperature limits based on evaporative flux

following discussion. Considering that liquid metals may show temperature enhanced erosion yields already in the order of $10-20 \%$ for $\mathrm{Sn}[21]$ and $\mathrm{Li}[22]$ a more stringent level on evaporation down to or $10^{20} \mathrm{~m}^{-2} s^{-1}$ is also considered. Based on $[23,24,25]$

$$
\frac{p_{\text {Brems. }}}{p_{\alpha}} \sim 0.107 \frac{\left(1-f_{\text {Imp }} \cdot Z+f_{\text {Imp }} \cdot Z^{2}\right)}{\left(1-f_{\text {Imp }} \cdot Z\right)^{2}}
$$

one can estimate that $\mathrm{Sn}$ in contrast to $\mathrm{Li}$ will most likely face limits due to radiative losses similar to W [19]. A rough estimate shows however that a flux close to $10^{20}$ 
$\mathrm{m}^{-2} s^{-1}$ can be tolerated even for Sn. Dedicated transport analysis linking the local source and the potential plasma impact by means of a transport model need to be performed. Under high-density plasma conditions in the divertor, re-deposition of lost material could help control the material loss and increase the operational temperature. Assuming the maximum allowed effective loss flux of $\Gamma_{\text {eff }} \sim 10^{20} \mathrm{~m}^{-2} s^{-1}$ figure 1(b) shows the evolution of the maximum allowable surface temperature with the actual evaporation rate linked to a given re-deposition efficiency for both $\mathrm{Li}$ and $\mathrm{Sn}$. For both, a significant re-deposition efficiency is required to extend the allowable surface temperature. Even though re-deposition of the material might aleviate the lifetime issue dedicated experiments establishing a power-balance under theses conditions are yet required.

In contrast to Li, Sn allows for comparably high surface temperatures due to low evaporation and material losses even when not considering re-deposition.

\section{The Capillary Porous System}

Induced currents can occur in a liquid metal sheet under tokamak conditions, especially during plasma disruptions. Decaying vertical fields or a moving liquid sheet itself will induce currents. Already for small tokamaks $\left(\mathrm{B}_{T} \sim 3 \mathrm{~T}, \mathrm{~B}_{\text {pol }} \sim 0.2 \mathrm{~T}\right)$ forces close to $10^{7} \mathrm{~N} \cdot \mathrm{m}^{-3}$ can occur [26]. As part of the application of liquid metals, stabilizing concepts have hence been proposed including the use of capillary action to counteract disruption and jxB forces. Typically a mesh or a porous substrate (fig. 2) on top of a actively

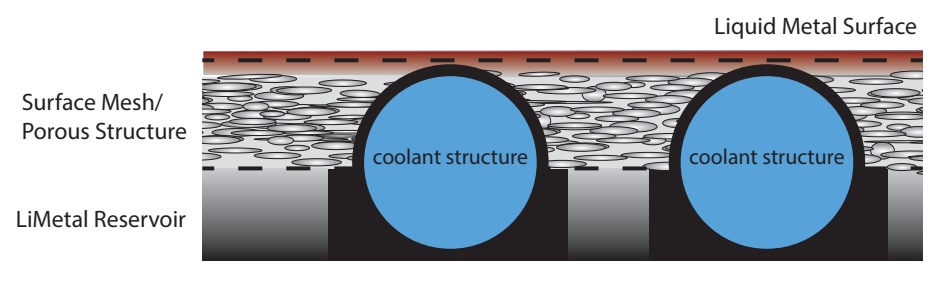

Figure 2: CPS Components

cooled structure together with a liquid metal reservoir is used in the so called Capillary Porous System (CPS) [15, 27, 16, 9, 13, 28, 12]. To facilitate capillary action, the open radius of the porous structure or mesh is typical in the sub-mm range $(10-200 \mu \mathrm{m})$. In addition lately the so called LIMIT system using thin metal trenches and thermoelectric forces has been proposed $[29,30]$. Due to the capillary force ideally a pressure difference along the capillary $\Delta P=\frac{2 \gamma \cos (\theta)}{r}$ is causing the rise of the liquid metal through the porous system : $h=\frac{2 \gamma \cos \theta}{\rho g r}$. Depending on the material properties and the size of the capillary structures significant replenishment of the liquid surfaces can be guaranteed as well as strong forces compensating against external de-stabilization, such as disruption forces. 
Despite having to choose different capillary radii, depending on the LM, one can establish capillary forces large enough to stabilize $\left(>5000 \mathrm{~N} / \mathrm{m}^{2}\right)$ and can also allow a capillary height comparable to any CPS structure size $(\sim \mathrm{mm} / \mathrm{cm})$.

An issue related to the replenisment of evaporative or transient losses is the wicking of such as structure, the actually possible maximum rate of material throughput. Initial indications [31] are that for all the metals discussed a porous size can be found to allow material throughput large enough for surface replenishment even including MHD effects.

\subsection{Material Choices}

With respect to applying a material for a CPS under fusion conditions the wetting between the two materials (liquid, solid) is as important as potential alloying, heat-flux resistance and nuclear safety aspects of the substrate. For the purpose of this work three substrate materials, Stainless Steel, $\mathrm{W}$ and molybdenum, next to the candidate materials $\mathrm{Sn}, \mathrm{Ga}$, and $\mathrm{Al}$ have been considered. Aluminum is however not discussed further due to long living activation products [32]. From laboratory experiments and

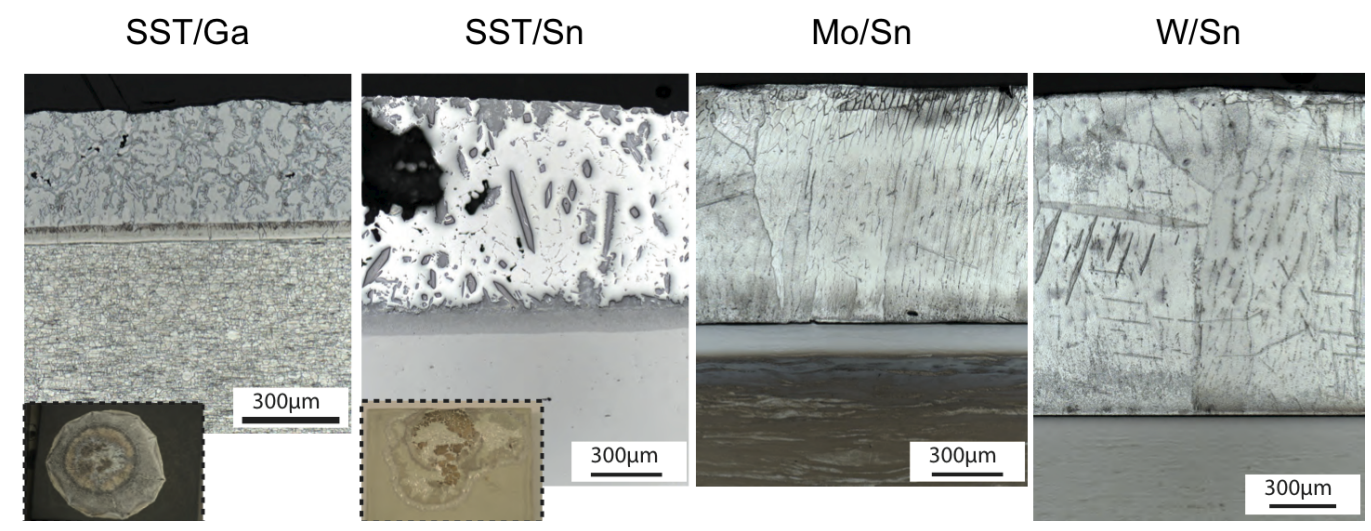

Figure 3: Micrographs of Material Cuts. The liquid metal on top of the substrate material as well as potential interaction and mixed zones are depicted.

literature data [33] one can establish potential viability of the material combinations proposed here. Figure 3 displays cuts through laboratory specimen of Stainless Steel (SST), W, Mo, and Ga, Sn respectively. As seen from [33] as well as from the SST/Ga combination Ga readily forms alloys with any of the substrate materials and hence faces issues when considering CPS system lifetime, while for the combination of Sn/Mo only a small interlayer is found. Sn shows no reaction to $\mathrm{W}$ with a very low $\mathrm{W}$ solubility even at elevated temperatures. (0.001 at\% $\mathrm{W}$ at $2273 \mathrm{~K}$ ) [33].

To study the actual wetting of the laboratory samples of SST, W and Mo were used to establish the wettability and contact angles between the respective materials. Under $10^{-6} \mathrm{mbar}$ the samples were heated up to $1260 \mathrm{~K}$. For all material combinations wetting, in many cases followed by reactions, was achieved at temperatures above 1000 K. For each of the substrate materials oxide layers are expected to hinder wetting. For 
Mo/Sn wetting starts only when preparing the sample by removing the oxide layer due to a heat treatment. In the case of $\mathrm{W} / \mathrm{Sn}$ wetting can only be achieved by facilitating the oxide layer removal by means of an hydrogen atmosphere as the oven cannot reach temperatures $(>1000 \mathrm{~K})$ required to decompose $\mathrm{W}$-oxides. In case of $\mathrm{W} / \mathrm{Sn}$ the contact angle is in the range of $\sim 40^{\circ}$, reducing the ideal capillary action by only a factor $\cos \left(40^{\circ}\right) / \cos \left(0^{\circ}\right)=0.77$.

\subsection{Transient Impact}

One major benefit of using liquid metals as a plasma facing material is its ability to replenish after transient induced losses. However the ability of the setup to handle transients depends also on the penetration of heat fluxes to the substrate material.

Based on [34] one can estimate the penetration of a heat pulse into the solid taking into account the parameters supplied in table 1.

For all materials considered here the heatwave assuming $\sim 1 \mathrm{GWm}^{-2}(1 \mathrm{~ms})$ is reaching values of below $1500 \mathrm{~K}$ already well in the first $1 \mathrm{~mm}$, meaning that choosing a design with a LM sheet of a few $100 \mu m$ should suffice to buffer transients. Obviously replenishment has to be in-line with the potential loss mechanisms.

\section{Heat Exhaust by CPS - RACLETTE Modeling}

In order to study the potential power-handling capabilities of a CPS based systems the RACLETTE (Rate Analysis Code for pLasma Energy Transfer Transient Evaluation) [35] code has been adapted for use with liquid armor materials. RACLETTE has previously been used to simulate damage to the ITER divertor monoblocks [36]. After having already been updated with the thermal properties of Li[37] RACLETTE had to be modified to allow next to transient evaporative events also steady state heat exhaust through a liquid target made up of $\mathrm{Li}, \mathrm{Sn}, \mathrm{Al}$, or Ga. Firstly the thermo-mechanical properties, e.g. for Sn [38, 39], were included, and secondly the behavior of RACLETTE modified. RACLETTE distinguishes between basically 4 domains, the coolant, the tubing, the initial armor and the top armor. Adapting RACLETTE allows to simulate the heat exhaust of a fully liquid metal sheet on top of a substrate material connected to an actively cooled substructure. For details of the actual modeling calculations and parameters refer to [35]. Figure 4 shows the steady state 4(a) and transient 4(b) impact of power onto the following PFCs configuration: $10 \mathrm{~mm}(\mathrm{Sn} / \mathrm{Li})$ on top of $4.4 \mathrm{~mm} \mathrm{~W}$ substrate, a $1.1 \mathrm{~mm} \mathrm{Cu}$ tube \& Water Coolant.

For figure 4(a) the maximum heat fluxes are chosen to allow the evolution into steady state conditions, without either evaporating the whole surface or exceeding the heat-exhaust capabilities of the coolant structure. For Sn a steady state heat flux of 25 $\mathrm{MW} / \mathrm{m}^{2}$ can be reached, while $10 \mathrm{MW} / \mathrm{m}^{2}$ is the maximum viable for $\mathrm{Li}$.

With the thick armor and its relatively low heat conductivity a strong evaporation

takes places in the early phases of the simulation $(<40 \mathrm{~s})$. Afterwards as most of the 


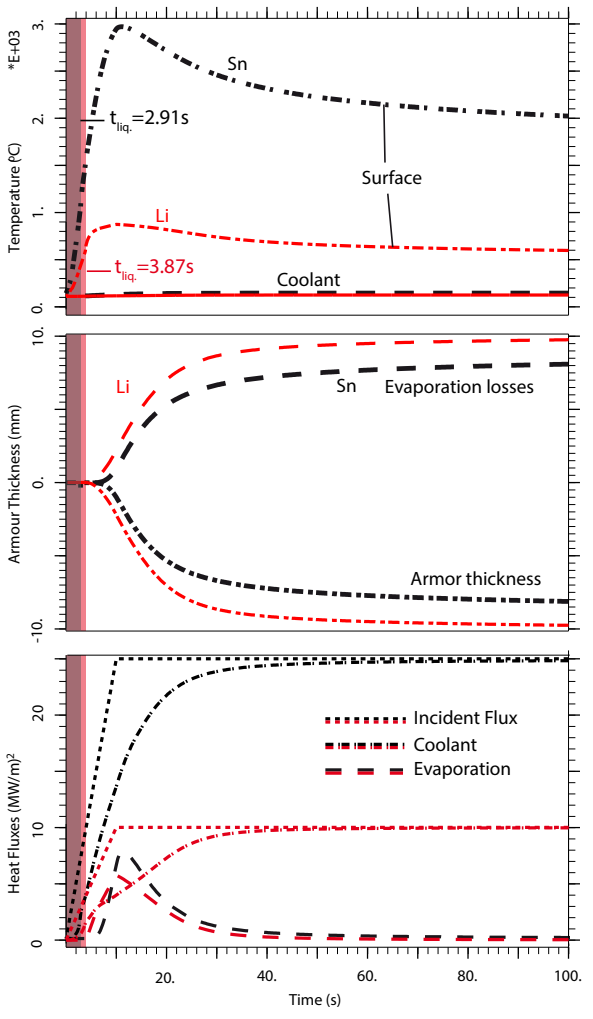

(a) Steady State
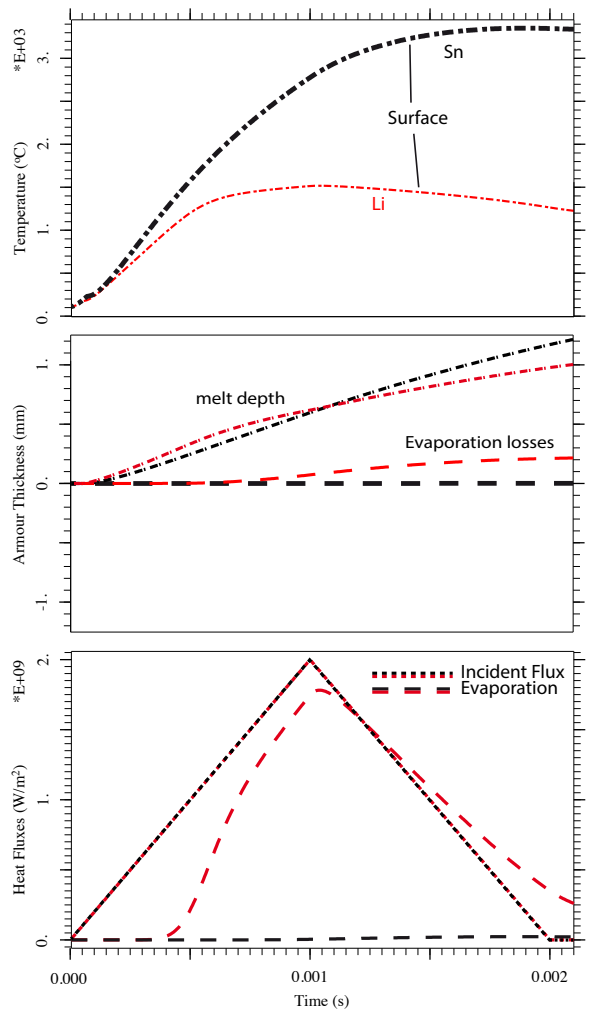

(b) Transient

Figure 4: RACLETTE Modeling

liquid metal is evaporated a thin film (1-3mm) on top of the structure remains now allowing for a direct heat conduction down to the coolant. All of the incident power is taken up by the cooling structure. This kind of configuration is envisioned for a CPS with minimal evaporative losses (cf fig. 4(a), tab. 2).

As discussed above the transient heat-exhaust and impact are one of the main area of liquid metal application 4(b) due to its replenishing mechanism. As transient heatfluxes can easily range in the $\mathrm{GW} / \mathrm{m}^{-2}$ range $[40,2]$ a value of $2 \mathrm{GWm}^{-2}$ and a duration of $2 \mathrm{~ms}$ has been chosen here. To show the penetration depth into the material the material was chosen to be solid at the start of the simulation. In contrast to the steady state simulation no heat is reaching the coolant as the heat wave is not penetrating through the material during only $2 \mathrm{~ms}$. Two main differences between Li and tin are clearly visible: Firstly, the maximum temperature reached for $\mathrm{Sn}$ is much higher due to its lower heat capacity while retaining the same amount of melting, and secondly despite the much lower surface temperature reached in the case of Li a significantly larger material loss due to evaporation is observed.

In both cases, steady state and transient heat-flux, Sn shows the potentially superior power-handling ability even when demanding low evaporation. This means potentially larger steady state abilities, but also less material losses during even larger transients. 
RACLETTE is yet missing a replenishment mechanism, the ability to simulate re-deposition and vapor-shielding as well as application of a porous mixed material.

\section{Plasma Exposures and Outlook}

Even though Sn shows promising results in terms of calculated limits experimental results are required to establish the actual viability.

For proof of principle exposures the linear plasma device PSI-2 [41, 42] at Juelich has been used to expose a Sn-wetted CPS-like structure to a linear plasma. In addition exposures at the PILOT-PSI facility have been performed.

Figure 5 displays the basic setup of the target manipulator as well as the dedicated liquid metal \& Mesh target construction. Five layers of a W mesh $\left(r_{\text {opening }}=0.2\right.$ $\mathrm{mm}, \mathrm{r}_{\text {fiber }}=0.05 \mathrm{~mm}$ ) are clamped by means of a TZM ring onto a TZM disc. The whole thickness of the $\mathrm{W}$ mesh structure is roughly $0.3 \mathrm{~mm}$. Even though the target manipulator is actively cooled the Sn samples, in particular the TZM disc, are clamped to a back plate with low thermal contact, to facilitate melting. One of the targets is

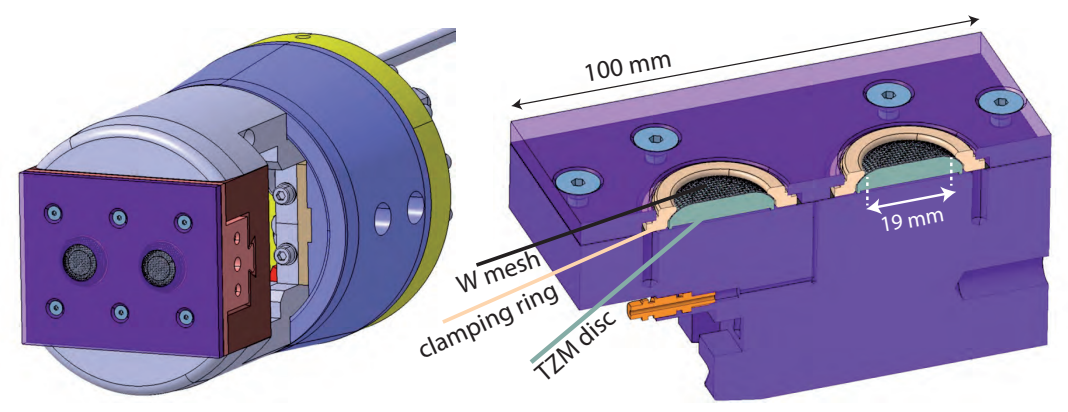

Figure 5: Setup for Plasma Exposure in PSI-2

filled by $1.13 \mathrm{~g}$ Sn while the other remains empty, exposing the bare W. The filling is done under $1 \mathrm{mbar}$ of $\mathrm{H}_{2}$ atmosphere similar to the wetting experiments. As expected the material is confined with in the mesh by the capillary forces. No material is moved onto the clamping ring due to gravity, despite the liquid Sn sample being vertically exposed.

The samples are exposed to a PSI-2 plasma with $\mathrm{n}_{e}=7.6 \cdot 10^{11} \mathrm{~cm}^{-3} \mathrm{~T}_{e}=9 \mathrm{eV}$, Ionflux $=4.8 \cdot 10^{17} \mathrm{~cm}^{-2} \mathrm{~s}^{-1}$. With the samples being exposed in total 1 hour $(30 \mathrm{~min}+$ $15 \mathrm{~min}+15 \mathrm{~min}$ ) the maximum surface temperature reached was $1173 \mathrm{~K}$. The flattop in temperature is reached typically after $800 \mathrm{~s}$. For the unexposed W mesh the impact of the heat-flux can clearly be seen, the mesh starts to glow, while the liquid Sn remains mostly unchanged. $\left(\epsilon_{S n} \sim 0.06, \epsilon_{W} \sim 0.3\right)$.

Despite the temperature measured being only $\sim 1173 \mathrm{~K}$ material in the amount of $0.24 \mathrm{~g}$ is lost as visible from fig. 6. The red encircled area shows an area already retaining less Sn then the other parts of the samples, the black area depicts the area suffering from mass loss during the PSI-2 exposures. From infrared images clear differences in the 


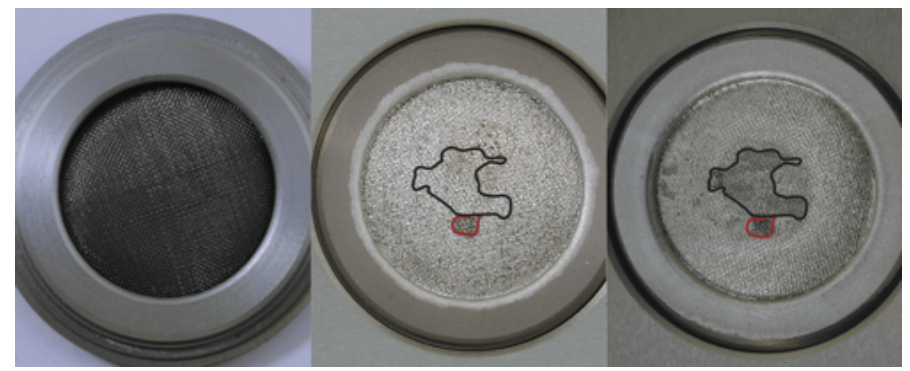

(a) CPS-like mesh strutter for PSI exposure, left to right: empty, before exposure, after exposure

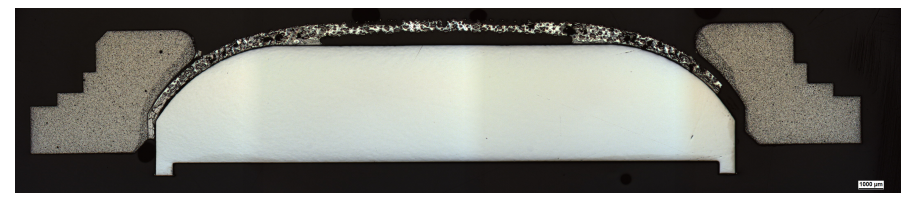

(b) Cut through sample, after exposure in PSI-2

Figure 6: CPS-like mesh strutter for PSI exposure, left to right: empty, before exposure, after exposure

behavior of the non-wetted area can be observed, similar to the area where material is lost. From flux calculations one can attain that having areas with only $100 \mathrm{~K}$ higher then the average temperatures would allow for the loss of the material by evaporation. This means different wetting of some areas of the samples exposed and potentially different heat-conduction within the mesh to substrate setup cause local evaporation.

Figure 6(b) shows a cut through the material after exposure, here clearly the void between wetted mesh and TZM substrate disc is visible. On the top surface hence a whole layer of mesh is bare after exposure due to material loss.

Experiments at Pilot-PSI have already successfully started looking at the issue of temperature enhanced erosion. A recessed molybdenum target was filled with Sn and covered with a molybdenum mesh of $0.44 \mathrm{~mm}$ aperture to keep the liquid metal in the reservoir. The Liquid Sn was heated to around $\sim 1073 \mathrm{~K}$ to wet the mesh. This target was then heated in the linear plasma device Pilot-PSI with an Argon plasma at a cathode current of $110 \mathrm{~A}$ and magnetic field of $0.4 \mathrm{~T}$, while scanning the bias down to $-40 \mathrm{~V}$ negative bias to increase the incoming ion energy. The target temperature was measured with an IR-camera cross calibrated with a pyro-spectrometer to give an emissivity of 0.06. The $\mathrm{Sn}(\mathrm{I})$-emission at $452.5 \mathrm{~nm}$ was measured and is assumed to be linearly proportional to the amount of Sn eroded into the plasma. As can be seen from the fig. 7, after a slow linear increase of emission a rapid exponential increase with respect to target temperature starts at around $1273 \mathrm{~K}$. The fact that this increase depends so much on bias energy indicates that this rapid increase cant be explained by evaporation alone. At startup there was magnetic field without bias for a short time, which explains the low emission up to $473 \mathrm{~K}$. The large deviations at 1173-1273 K, especially clear for the $-40 \mathrm{~V}$ curve, are due to Sn-droplets migrating trough the view of 


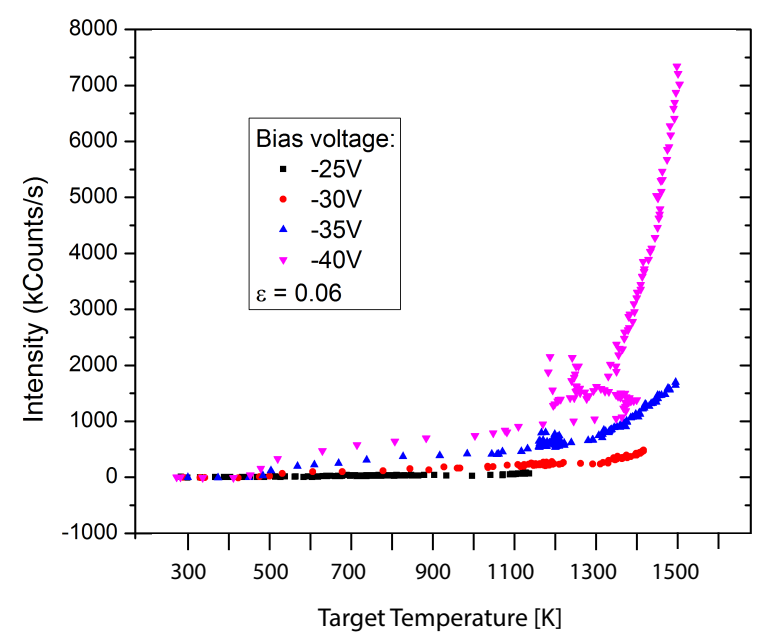

Figure 7: Tin intensity versus surface temperature

the spectrometer. For future experiments in the TEC facilities Pilot-PSI, Magnum-PSI and PSI-2 three main aspects of liquid metals are to be studied. Firstly the samples in PSI-2 will be made heatable to allow controlled temperature exposures especially with the focus on studying further the temperature enhanced erosion of Sn [21], in collaboration with Pilot-PSI at its higher steady state heatflux capabilities. Studying the erosion up to the limits set by evaporation is vital to understand the particle loss mechanisms. Secondly the fuel retention of liquid metals especially Li and Sn is highly uncertain and needs to be elaborated under controlled conditions. Tin as it doesn't readily form a stable hydrid may be less of an issue $[4,38,39]$. Thirdly, the heatexhaust of Sn under steady state and transient conditions needs to elaborated. For PSI-2 transient tests are envisioned by means of leaser heating, while for steady state heat exhaust a dedicated actively cooled design for MAGNUM-PSI is envisioned (cf. fig $2)$.

\section{Conclusions}

From the results presented here one can draw the following conclusions based on the calculations as well as the preliminary experiments. A stabilizing concept similar to the CPS or LIMITs concept is certainly required to allow for operation of a liquid metal under tokamak conditions. Despite Li being the favorite material up to now, it is reasonable to explore $\mathrm{Sn}$ as a most likely alternative liquid metal. Especially when considering low evaporation scenarios Sn has clear benefits without having to rely on strong re-deposition. A main benefit of liquid metals is their ability for self-healing and replenishment after transients. Allowing for evaporation of thin surface layers without an impact on the substrate lifetime of the PFCs is highly superior to solid materials. Steady state power-handling, even though in case of Sn potentially superior to solid materials, would not necessarily be the main argument for using liquid metals. Experiments both 
on Li and Sn, especially with respect to their Plasma-Wall-Interaction properties such as sputtering and fuel retention next to steady state exhaust, need still to be performed in order to establish theses liquid metals as viable reactor option.

\section{Acknowledgements}

This work, supported by the European Communities under the contract of Association between EURATOM/FZJ, was carried out within the framework of the EFDA Task Force on Plasma-Wall Interactions. The views and opinions expressed herein do not necessarily reflect those of the European Commission. Metallography images are presented thank to the collaboration with our colleagues from IEK-2.

[1] Pitts, R. et al. Journal of Nuclear Materials, 415 (2011), 1 SUPPL, - .

[2] Pitts, R. et al. Journal of Nuclear Materials, (2013), -. Article in Press.

[3] Mattas, R. et al. Fusion Engineering and Design, 49-50 (2000), 0, 127 - 134.

[4] Brooks, J. e. a. Fusion Science and Technology, 47 (2005), 669-677.

[5] Mohamed, A. and Abdou. Fusion Engineering and Design, 72 (2004), 1-3, 1 - 2. Special Issue on Innovative High-Power Density Concepts for Fusion Plasma Chambers.

[6] Nygren, R. et al. Fusion Engineering and Design, 72 (2004), 1-3, 223 - 244. Special Issue on Innovative High-Power Density Concepts for Fusion Plasma Chambers.

[7] Mansfield, D. et al. Nuclear Fusion, 41 (2001), 12, 1823.

[8] Majeski, R. et al. Fusion Engineering and Design, 72 (2004), 1-3, 121 - 132. Special Issue on Innovative High-Power Density Concepts for Fusion Plasma Chambers.

[9] Tabares, F. et al. Problems of Atomic Science and Technology, - (2008), 6, 3-7.

[10] $\mathrm{Hu}$, J. et al. Fusion Engineering and Design, 85 (2010), 6, 930 - 934. Proceedings of the 1st International Workshop on Lithium Applications for the Boundary Control in Fusion Devices.

[11] Zuo, G. et al. Journal of Nuclear Materials, 415 (2011), 1, Supplement, S1062 - S1066. Proceedings of the 19th International Conference on Plasma-Surface Interactions in Controlled Fusion.

[12] Mirnov, S. et al. Nuclear Fusion, 51 (2011), 7, 073044.

[13] Apicella, M. et al. Journal of Nuclear Materials, 386-388 (2009), 821-823.

[14] Kugel, H. et al. Fusion Engineering and Design, (2011), 0,-.

[15] Evtikhin, V. et al. Fusion Engineering and Design, 49-50 (2000), 0, 195 - 199.

[16] Evtikhin, V. et al. Journal of Nuclear Materials, 307-311, Part 2 (2002), 0, $1664-1669$.

[17] Heiland, W. et al. Taschenbuch fr Chemiker und Physiker. Springer (1992).

[18] Stangeby, P. The plasma boundary of magnetic fusion devices. IoP (2000).

[19] Puetterich, T. et al. Nuclear Fusion, 50 (2010), 2, 025012.

[20] Eckstein, W. et al. Sputtering data. Technical Report IPP 9/82, Max-Planck-Instut fuer Plasmaphysik (1993).

[21] Coventry, M.; Allain, J. and Ruzic, D. Journal of Nuclear Materials, 335 (2004), 1, 115 - 120.

[22] Allain, J. P.; Coventry, M. D. and Ruzic, D. N. Phys. Rev. B, 76 (2007), 205434.

[23] Jensen, R. et al. Nuclear Fusion, 17 (1977), 6, 1187.

[24] Post, D. et al. Physics of Plasmas, 2 (1995), 6, 2328-2336.

[25] Scavino, E. Transport of Laser-Ablated Impurities in TCV. Ph.D. thesis, Ecole Polytechnique Fdrale de Lausanne (2003). LRP 758/03.

[26] Winter, J. et al. Fluid targets for heat removal in fusion reactors. Technical report, Forschungszentrum Jülich (1994).

[27] Evtikhin, V. et al. Fusion Engineering and Design, 56-57 (2001), 0, 363 - 367.

[28] Mirnov, S. Journal of Nuclear Materials, 390-391 (2009), 876-885. 
[29] Jaworski, M.; Morley, N. and Ruzic, D. Journal of Nuclear Materials, 390-391 (2009), 1055-1058.

[30] Jaworski, M. et al. Journal of Nuclear Materials, 415 (2011), 1, Supplement, S985 - S988. Proceedings of the 19th International Conference on Plasma-Surface Interactions in Controlled Fusion.

[31] Jaworski et al, M. (2012). 1oth European Fusion Physics Workshop (EFPW), Ericeira , Portugal.

[32] Gilbert, M. and Forrest, R. Handbook of activation data calculated using easy-2003. UKAEA FUS 509, EURATOM/UKAEA Fusion Association (July 2004).

[33] Springer MMaterial: The Landolt-Boernstein Database. Springer (2013). Http://www.springermaterials.com/docs/index.html.

[34] Carslaw, H. and Jaeger, J. Heat Conduction in Solids. Oxford at the Clarendon Press (1959).

[35] Raffray, A. and Federici, G. Journal of Nuclear Materials, 244 (1997), 2, 85-100.

[36] Federici, G. and Raffray, A. Journal of Nuclear Materials, 244 (1997), 2, 101-130.

[37] Davison, H. Compilation of thermophysical properties of liquid lithium. Technical report, National Aeronautics and Space Administration, Lewis Research Center, Cleveland Ohia (1968).

[38] Sharafat, S. and Ghoniem, N. Summary of thermo-physical properties of sn, and compounds of sn-h, sn-o, sn-c, sn-li, sn-si and comparison of properties of sn, sn-li, li, pb-li. Technical report, University of California, Los Angeles (2000). UCLA-UCMEP-00-31.

[39] Culpin, M. F. Proceedings of the Physical Society. Section B, 70 (1957), 11, 1069.

[40] Mitteau, R. et al. Physica Scripta, 2011 (2011), T145, 014081.

[41] Kastelewicz, H. and Fussmann, G. Contributions to Plasma Physics, 44 (2004), 4, 352-360.

[42] Unterberg, B. et al. Fusion Engineering and Design, 86 (2011), 9-11, 1797-1800. 